\title{
Emergency thromboembolectomy for a massive saddle embolism crossing an atrial septal defect
}

\author{
Matthew A. Warner, MD • Noman E. Torres, MD - Seri A. Carney, MD • \\ Michael E. Lowe, MD • Rochelle J. Molitor, MD
}

Received: 11 February 2017/Revised: 15 February 2017/ Accepted: 23 February 2017/Published online: 2 March 2017

(C) Canadian Anesthesiologists' Society 2017

Acute ischemic stroke is often complicated by hemorrhagic transformation that may preclude pharmacologic venous thromboembolic prophylaxis. ${ }^{1}$ The risk of thrombotic complications after acute stroke in the absence of pharmacologic prophylaxis is substantial, with the incidence of deep venous thrombosis reported at 50\% and that of clinically apparent pulmonary embolism at 10 $13 \% .^{2,3}$

We report the case of a 52-yr-old woman who suffered an acute right middle cerebral artery ischemic stroke with subsequent hemorrhagic transformation that required decompressive craniectomy. She was discharged from the hospital two weeks later on aspirin $81 \mathrm{mg}$ for secondary stroke prevention. Approximately four weeks later, the patient was readmitted to the hospital following a syncopal event. She was markedly hypoxemic and hypotensive. Computed tomography revealed an acute pulmonary saddle

Electronic supplementary material The online version of this article (doi:10.1007/s12630-017-0858-4) contains supplementary material, which is available to authorized users.

M. A. Warner, MD ( $\square)$

Department of Anesthesiology, Division of Critical Care

Medicine, Mayo Clinic, Rochester, MN, USA

e-mail: warner.matthew@mayo.edu

\section{N. E. Torres, MD}

Department of Anesthesiology, Division of Adult Cardiothoracic Anesthesiology, Mayo Clinic, Rochester, MN, USA

S. A. Carney, MD

Anesthesia Physicians, Ltd., Sioux Falls, SD, USA

M. E. Lowe, MD - R. J. Molitor, MD

Department of Anesthesiology, Anesthesiology Residency

Program, Mayo Clinic, Rochester, MN, USA embolism. She was emergently transferred to the operating room for open thromboembolectomy under cardiopulmonary bypass. Intraoperative transesophageal echocardiography (Figure, supplemental video) revealed thrombus migration from the right atrium into the left atrium through an atrial septal defect, as well as severe right ventricular enlargement and dysfunction. The surgical specimen, measuring $45 \mathrm{~cm}$ in length, was extracted from its origin at the junction of the inferior vena cava and right atrium where it then folded on itself and crossed through the atrial septal defect before extending into the right ventricle, the right ventricular outflow tract, and both pulmonary arteries. Surgical extraction was associated with rapid improvements in circulatory hemodynamics and oxygenation.

Massive pulmonary embolus requiring open thromboembolectomy is rare, typically limited to those with absolute contraindications to lytic therapy (i.e., recent intracranial hemorrhage). In this case, the patient had a very large thrombus at heightened risk for paradoxical embolization given the presence of an atrial septal defect. After consultation with our colleagues in neurological surgery, neurology, vascular medicine, and cardiovascular surgery, the benefits of open thromboembolectomy were deemed to outweigh the potential risk of recurrent intracranial hemorrhage associated with the systemic administration of unfractionated heparin for cardiopulmonary bypass.

Optimal perioperative hemodynamic management during open pulmonary thromboembolectomy is critical for maximizing the chances of a positive outcome. Anesthetic goals are centered on support of the failing right ventricle, including maintaining systemic vascular resistance and coronary perfusion pressure, supporting 

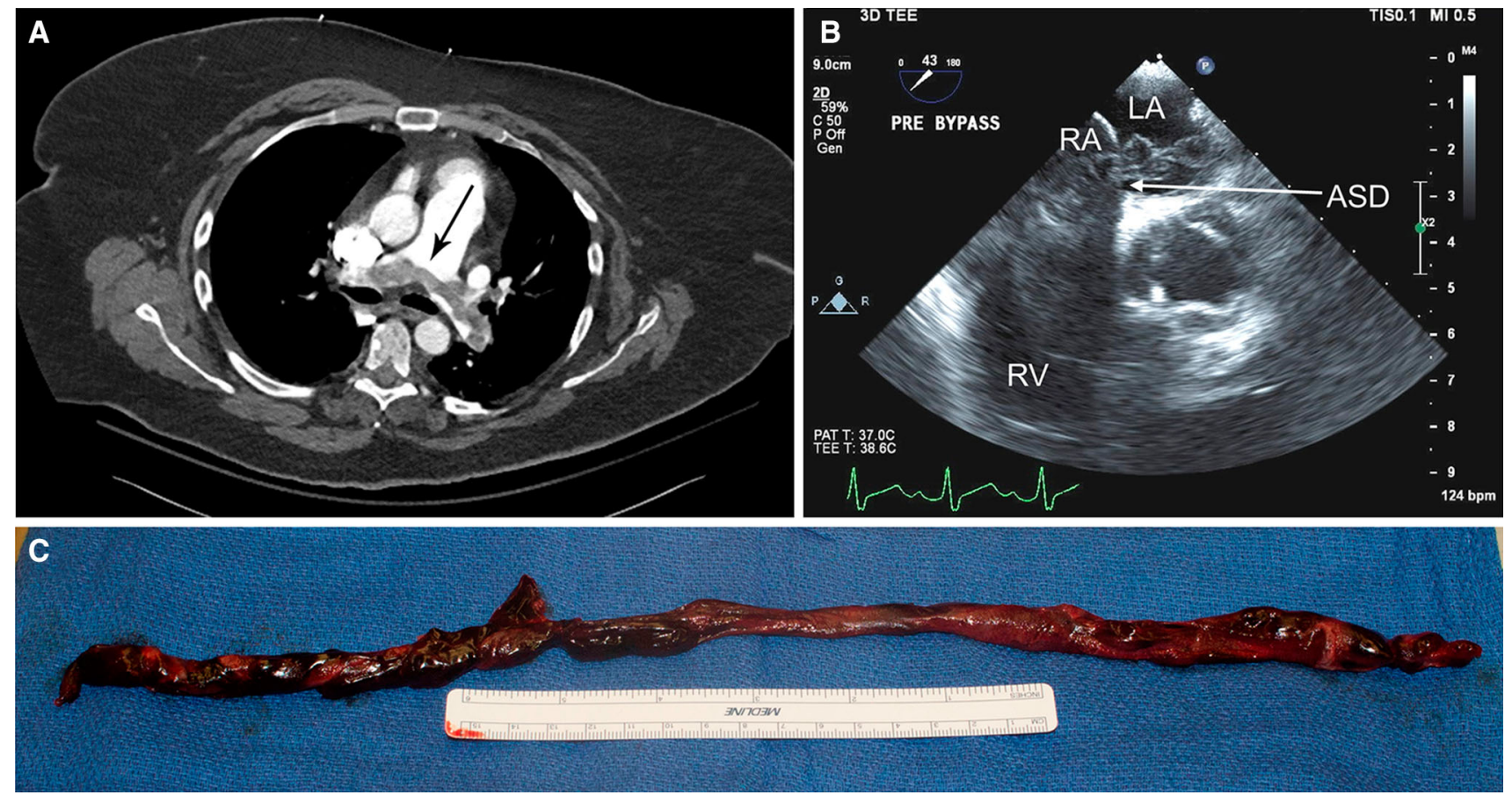

Figure Acute pulmonary saddle embolism as revealed by a "fillingdefect" on contrast-enhanced computed tomography imaging (panel A). Intraoperative transesophageal echocardiography imaging (panel $\mathrm{B}$, supplemental video) revealed thrombus migration from the right atrium (RA) into the left atrium (LA) through an atrial septal defect

ventricular inotropy and the heart rate, and minimizing additional increases in pulmonary vascular resistance. ${ }^{4}$ In this case, meticulous titration of intravenous epinephrine, norepinephrine, and vasopressin along with judicious fluid administration and avoidance of hypoxemia and hypercarbia during induction and maintenance of anesthesia resulted in adequate hemodynamic stability. Fortunately, this patient improved rapidly following thromboembolectomy and suffered no bleeding complications associated with systemic heparinization. An infrarenal inferior vena cava filter was placed on postoperative day 1 to prevent recurrent thromboembolic episodes. She was discharged home on postoperative day 6 .

Financial support \& conflicts of interests None.

Editorial responsibility This submission was handled by Dr. Hilary P. Grocott, Editor-in-Chief, Canadian Journal of Anesthesia.
(ASD) before folding back on itself and extending into the right ventricular outflow tract, causing right ventricular (RV) enlargement and dysfunction. The surgical specimen (panel C) measured $45 \mathrm{~cm}$ in length and was extracted in its entirety followed by rapid clinical improvement

\section{References}

1. Sussman ES, Connolly ES Jr. Hemorrhagic transformation: a review of the rate of hemorrhage in the major clinical trials of acute ischemic stroke. Front Neurol 2013; 4: 69.

2. Kelly J, Rudd A, Lewis R, Hunt BJ. Venous thromboembolism after acute stroke. Stroke 2001; 32: 262-7.

3. Brandstater ME, Roth EJ, Siebens HC. Venous thromboembolism in stroke: literature review and implications for clinical practice. Arch Phys Med Rehabil 1992; 73: S379-91.

4. Rosenberger $P$, Shernan SK, Shekar PS, et al. Acute hemodynamic collapse after induction of general anesthesia for emergent pulmonary embolectomy. Anesth Analg 2006; 102: 1311-5. 